\title{
Recent Insights into the Cell Biology of Bladder Smooth Muscle
}

\author{
Rosalyn M. Adam \\ Urological Diseases Research Center, Department of Urology, Children's Hospital Boston and Department of \\ Surgery, Harvard Medical School, Boston, Mass., USA
}

\section{Key Words}

Smooth muscle - Growth factors - Signal transduction • Phenotypic modulation

\begin{abstract}
Much of current biomedical research is focused on the development of 'targeted therapies' based on detailed knowledge about the signals that mediate aberrant cellular behavior in a given disease. Although this concept has been used most widely in cancer treatment, the same strategy applies to nonmalignant conditions such as pathologic tissue expansion in the genitourinary tract. A rigorous understanding of the key molecular events and pathways that underlie normal and pathologic activity of the bladder would allow us to identify potential targets for rational drug design. In this review, I will summarize our current understanding of cell signaling in bladder smooth muscle and highlight potential targets for drug-based treatment of tissue remodeling in the lower urinary tract.
\end{abstract}

Copyright $(\subset) 2006$ S. Karger AG, Basel

\section{Introduction}

Alterations in bladder smooth muscle growth and contractility underlie a number of pathologies of the lower urinary tract, including hypertrophic bladder growth sec- ondary to outlet obstruction and detrusor overactivity. Although the macroscopic changes that occur in the bladder wall exposed to pathologic stimulation have been appreciated for many years, the signals that underlie tissue remodeling at the molecular level are still poorly understood. As part of the normal cycling between full and empty states, the bladder wall is exposed to global mechanical and neural inputs, as well as local stimulation from growth factors, cytokines and the extracellular matrix (ECM). Aberrant activity of any of these stimuli leads to structural and functional changes that promote not only bladder dysfunction, but upper urinary tract damage as well. This review will focus on recent advances in our understanding of the effects of bladder smooth muscle stimulation on intracellular signaling (fig. 1a), and how these events may be targeted therapeutically (fig. 1b).

\section{Stretch-Stimulated Signaling in Bladder Smooth Muscle}

The tissues that comprise the bladder wall are continually exposed to mechanical stimulation, during filling and emptying. The process of information transfer in cells exposed to mechanical signals is termed mechanotransduction [1]. Forces impinging on the cell surface are transmitted through discrete transducers in the plasma membrane and cytoskeleton to the cytoplasm and nucleus to

\section{KARGER \\ Fax +4161306 1234 E-Mail karger@karger.ch} www.karger.com
(C) 2006 S. Karger AG, Basel $1660-2129 / 06 / 1021-0001 \$ 23.50 / 0$

Accessible online at:

www.karger.com/nee
Rosalyn M. Adam, PhD

Enders Research Laboratories, Rm 1077

Children's Hospital Boston, 300 Longwood Ave.

Boston, MA 02115 (USA)

Tel. +1 617919 2019, Fax +1 617730 0248, E-Mail rosalyn.adam@childrens.harvard.edu 


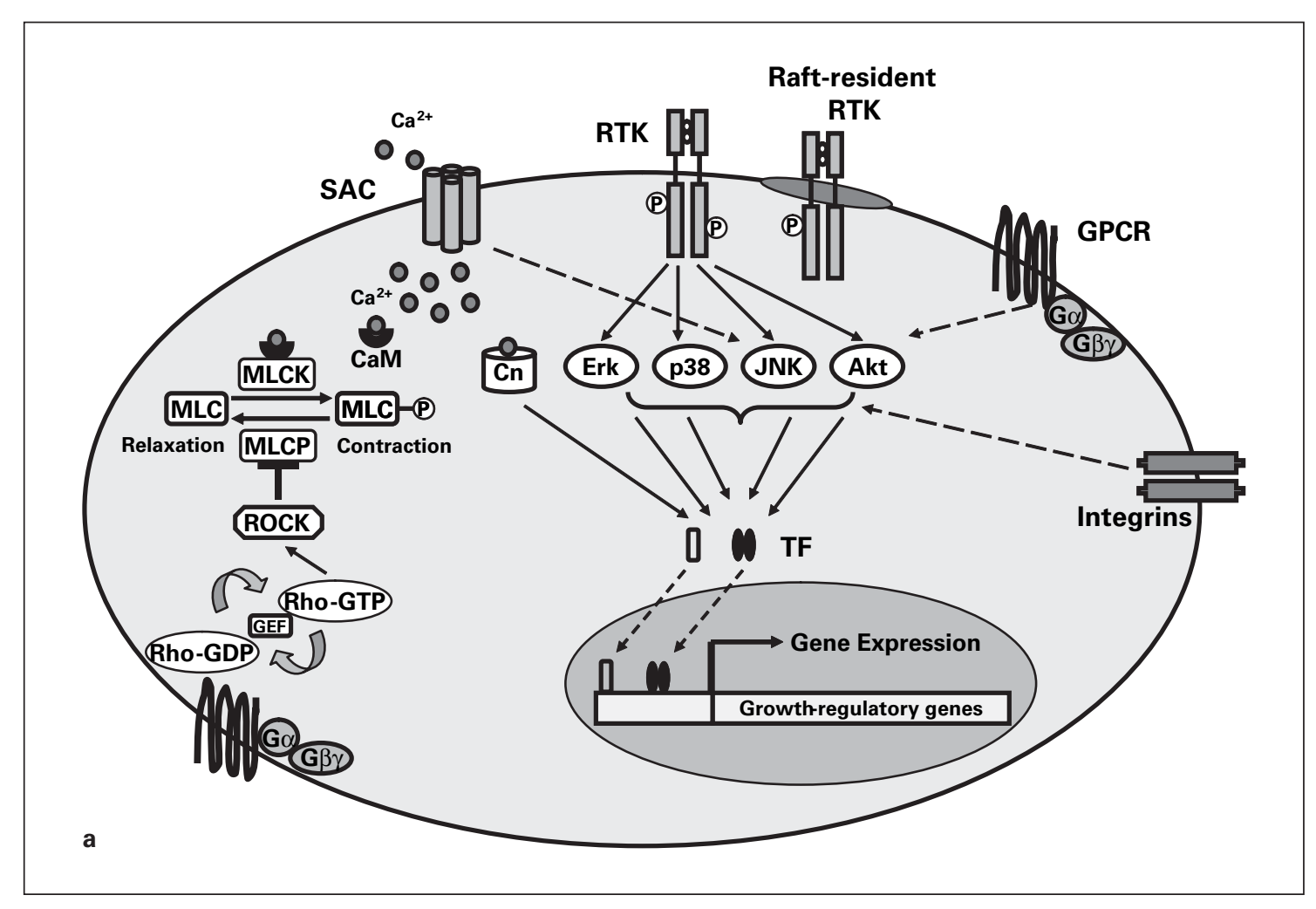

Fig. 1. Signaling cascades in bladder SMC. a External stimuli are transduced by stretch-activated ion channels (SAC), receptor tyrosine kinases (RTK), G-protein-coupled receptors (GPCR) and integrin subunits. SAC-mediated calcium $\left(\mathrm{Ca}^{2+}\right)$ entry promotes calmodulin $(\mathrm{CaM})$ binding and activation of myosin light chain kinase (MLCK), leading to MLC phosphorylation and initiation of smooth muscle contraction. MLC phosphatase (MLCP)-induced relaxation is inhibited by Rho-associated kinase (ROCK). ROCK is activated by Rho-GTP. $\mathrm{Ca}^{2+}$ influx also activates calcineurin $(\mathrm{Cn})$, leading to dephosphorylation and nuclear translocation of transcription factors (TF). RTK activation in response to ligand binding or mechanical stimuli promotes activation of the mitogen-activated protein kinases (MAPKs) Erk, p38 stress-activated protein kinase 2 (SAPK2) and c-jun N-terminal kinase (JNK), and Akt. These signaling intermediates in turn promote activation and nuclear translocation of TF, and alterations in gene expression. Activation of GPCRs also stimulates kinase cascades.

effect changes in gene and protein expression. Stretch stimulation has been shown to upregulate DNA synthesis and gene expression in bladder smooth muscle cells (SMC) through the activation of multiple parallel kinase cascades including the mitogen-activated protein kinases (MAPK) [extracellular signal regulated kinase (Erk); cJun N-terminal kinase (JNK); p38 stress-activated protein kinase 2 (p38SAPK2)] [reviewed in ref. 2], and the phosphoinositide-3-kinase (PI3K)/Akt pathway [3]. Signal transduction induced by mechanical stimulation of bladder SMC was reviewed recently [2], and will not be considered in extensive detail here.

To define a mechanistic link between stretch stimulation and growth of bladder SMC, investigators have evaluated expression of known SMC mitogens and other growth-regulatory proteins using a combination of in vitro, in vivo and ex vivo models of mechanical stimulation. Collectively, these studies have identified several stretch-regulated genes including insulin-like growth factor-1, heparin-binding EGF-like growth factor, nerve growth factor, COX-2 and the cysteine-rich protein Cyr61 $[2,4]$. A recent genome-wide analysis of gene expression in human bladder SMC exposed to cyclic stretch-relaxation in vitro showed that less than $0.2 \%$ of the expressed genome in bladder SMC was mechanically responsive [5], consistent with a highly selective cellular response to mechanical stimulation.

Cell surface receptors are also activated by mechanical forces. Nguyen et al. [6] demonstrated rapid activation of the epidermal growth factor receptor (EGFR)-related re- 


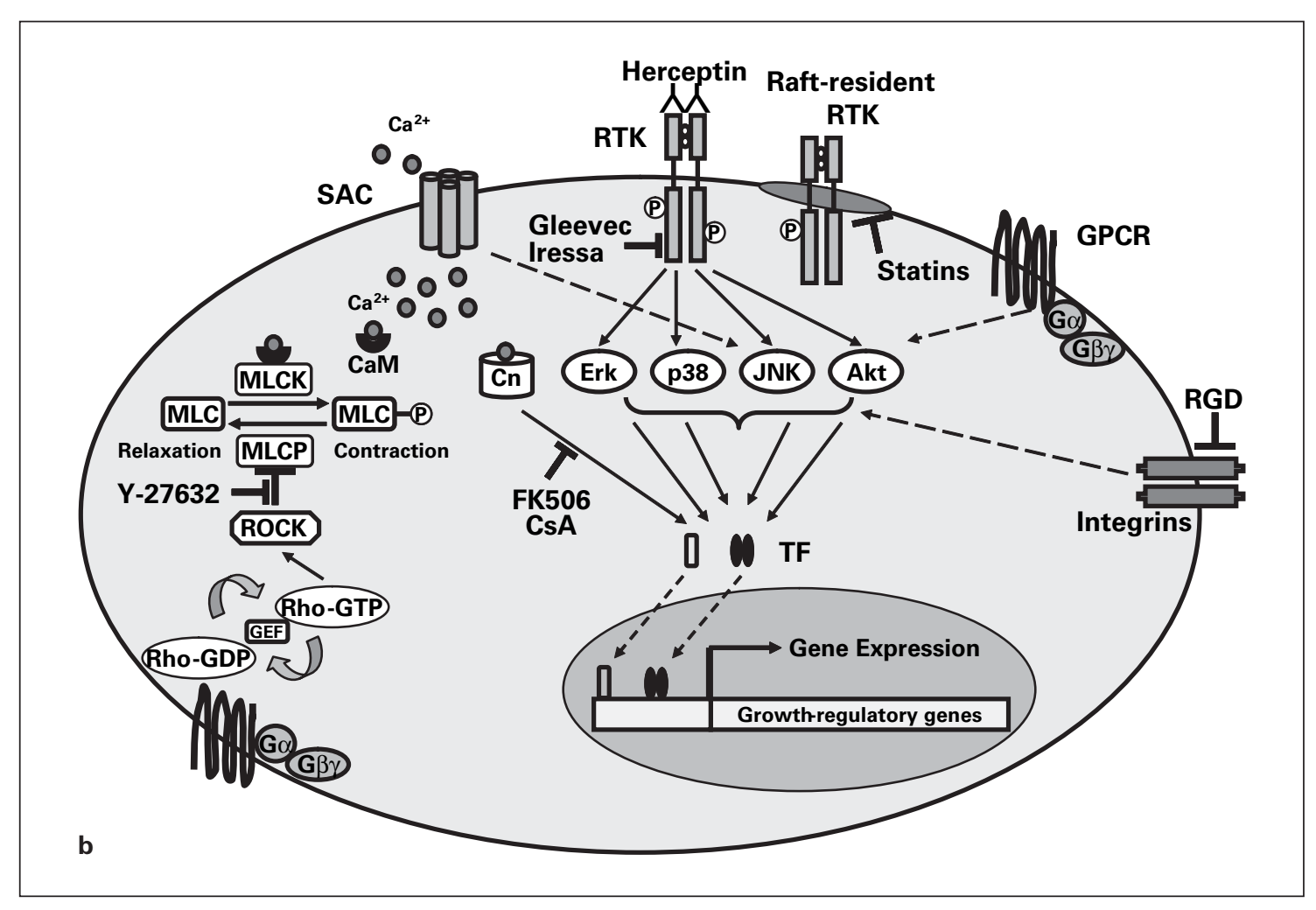

Fig. 1. b Signaling in bladder SMC can potentially be targeted with selective inhibitors. The pyridine derivative Y-27632 inhibits ROCK activity, thereby promoting smooth muscle relaxation. The immunosuppressive drugs, FK506 and cyclosporin A (CsA) target calcineurin. Receptor tyrosine kinase (RTK) activation is inhibited by antibody-based means (e.g. Herceptin), or by attenuation of intrinsic receptor kinase activity (e.g. Iressa or Gleevec). The activity of certain RTKs requires translocation to cholesterol-enriched lipid raft microdomains. Rafts can be ablated by inhibition of endogenous cholesterol synthesis using statin drugs that target 3-hydroxy-3 methylglutaryl coenzyme A (HMG-CoA) reductase, a key enzyme in cholesterol synthesis. Membrane targeting of Rho family members, required for their activation by guanine-nucleotide exchange, is also inhibited by statins. Signaling through integrins can be blocked with arginine-glycine-aspartic acid (RGD) peptides. GEF = Guanine-nucleotide exchange factor.

ceptor ErbB2 in bladder SMC exposed to stretch in vitro, and also implicated signaling through the angiotensin II type I receptor in stretch-stimulated gene expression The platelet-derived growth factor (PDGF) signaling axis has also been identified as a potent activator of DNA synthesis in bladder SMC $[3,7]$ In a comparative analysis, stretch and PDGF were both found to activate the PI3K/ Akt and p38 SAPK2 cascades in bladder SMC, and DNA synthesis induced by both agonists was attenuated in the presence of pharmacologic inhibitors of PI3K and p38 [3]. Although the expression of PDGF in the urinary tract remains undefined, studies of vascular SMC indicate that the PDGF receptor can be activated by mechanical stimuli in a ligand-independent manner [8]. Consistent with these observations, recent unpublished data from our lab- oratory suggest that stretch-stimulated Akt phosphorylation can be inhibited by pharmacologic blockade of the PDGFR. Therefore signaling through the PDGFR axis is likely to be physiologically relevant in bladder smooth muscle.

\section{Targeting Kinase Signaling for Therapeutic Gain}

The ability of receptor tyrosine kinases (RTK) to function as potent oncoproteins has led to several attempts to target these proteins therapeutically. RTK-targeted drugs in current clinical use for treatment of cancer include the humanized anti-erbB2 antibody, Herceptin (trastuzu- 
mab), the EGFR tyrosine kinase inhibitor Iressa (gefitinib) and Gleevec (imatinib mesylate) [9]. The original goal in developing Gleevec was to inhibit the PDGFR kinase. However, the drug was found to target multiple kinases, including the fusion protein Bcr-Abl and the RTK c-Kit such that Gleevec can be used to treat different types of cancer that are characterized by deranged activity of a specific kinase, e.g. gastrointestinal stromal tumors (c-kit activation) and chronic myelogenous leukaemia (Abl hyperactivity). The development of Herceptin, Iressa and Gleevec grew out of extensive knowledge about the signaling networks underlying tumor cell survival and proliferation, and the identification of specific molecular targets. Recent studies suggest a role for such agents in nonmalignant conditions with aberrant tissue growth. In support of this, Gleevec was found to attenuate spontaneous action potentials and contractions of smooth muscle strips from guinea pig detrusor [10]. Collectively, these observations suggest a potential role for kinase inhibitors for treatment of aberrant growth and contractility of bladder muscle.

\section{Signals That Regulate Smooth Muscle Contractility}

Alterations in bladder smooth muscle compliance and contractility underlie various disorders of the lower urinary tract. Smooth muscle contraction is activated by $\mathrm{Ca}^{2+}$-calmodulin-mediated activation of myosin light chain (MLC) kinase and subsequent phosphorylation of MLC to elicit contraction. The process is initiated by an increase in intracellular $\mathrm{Ca}^{2+}$ resulting from adrenergic receptor-stimulated release of $\mathrm{Ca}^{2+}$ from intracellular stores. Recent studies, however, have identified the small GTPase Rho and its effector Rho-associated kinase (ROCK) as mediators of smooth muscle contractility that act independently of changes in $\mathrm{Ca}^{2+}[11]$. ROCK phosphorylates and inactivates the myosin light chain phosphatase (MLCP) preventing dephosphorylation of MLC and promoting sustained smooth muscle contraction [11]. Activation of the Rho-ROCK axis has been demonstrated in models of bladder hypertrophy and in isolated bladder muscle strips [12, 13]. In addition, Rho has been proposed to regulate the differentiation status of SMC through its effects on actin polymerization and the transcription factor serum response factor (SRF) [14]. Although SRF is expressed ubiquitously, it regulates smooth muscle-specific gene expression as part of a complex with myocardin by binding to so-called CArG elements in the promoters of SM-restricted genes e.g. SM-myosin heavy chain (SM-MHC), $\alpha$-SM-actin and SM22 $\alpha$ [15]. Changes in cell shape that impinge on the cytoskeleton can therefore directly affect the differentiation status of SMC through actin-mediated effects on SRF-induced SM-specific gene expression.

Because of its central role in regulating smooth muscle contraction, ROCK represents a potential molecular target for lower urinary tract dysfunction resulting from bladder smooth muscle hypercontractility. The pyridine derivative Y-27632 was identified as a potent relaxant for vascular smooth muscle, that acts by inhibiting $\mathrm{Ca}^{2+}$ sensitization [16]. Several recent studies have demonstrated the ability of Y-27632 to inhibit agonist-induced contraction of bladder smooth muscle [12, 13]. Moreover, Rees et al. [17] also demonstrated a direct inhibitory effect of Y-27632 on proliferation of prostate SMC, suggesting that Y-27632 can target both aberrant smooth muscle contraction and growth.

A dual mode of action has also been demonstrated for doxazosin, an $\alpha_{1}$-selective adrenergic receptor blocker widely used to treat overactive bladder. Doxazosin was found to inhibit proliferation of human bladder SMC in culture [18]. Based on data obtained on other cell types, the anti-proliferative activity of doxazosin is believed to be independent of its anti-adrenergic effects since growth inhibition has been observed in cells lacking expression of adrenoceptors [19].

\section{Calcium Effects on Bladder Smooth Muscle}

Several recent reports have implicated changes in intracellular calcium in the activation of discrete signaling pathways in bladder SMC. Stretch-stimulated JNK activation in bladder SMC was attenuated in the presence of gadolinium chloride, an inhibitor of stretch-activated ion channels, but not by nifedipine or verapamil that target voltage-dependent $\mathrm{Ca}^{2+}$ channels [20]. JNK activation was also suppressed by W-7 and cyclosporin A, inhibitors of calmodulin and calcineurin, respectively [20]. Calcineurin is a calcium-dependent phosphatase that acts by dephosphorylating members of the NFAT transcription factor family. In the heart, hypophosphorylated NFAT translocates to the nucleus where it promotes activation of a hypertrophic gene expression program. Consistent with a pro-hypertrophic role for calcineurin, Nozaki et al. [21] recently demonstrated increased calcineurin expression in a mouse model of bladder hypertrophy secondary to outlet obstruction. Overexpression of the calcineurin 
catalytic subunit in bladder SMC increased cell size, with this hypertrophic effect reversed by the immunosuppressive drug, FK506 [21].

\section{Nitric Oxide in Bladder Smooth Muscle}

Bladder outlet obstruction also alters expression and activity of nitric oxide synthase (NOS) isoforms in the bladder. NOS converts $L$-arginine to nitric oxide (NO) which has multiple physiologic effects. These include vasodilatation and smooth muscle relaxation that result from NO-mediated activation of soluble guanylate cyclase and increased cyclic GMP (cGMP) levels [reviewed in ref. 22]. Bladder SMC have been shown to express inducible NOS (iNOS) and to release NO following stimulation with proinflammatory cytokines [23]. Exposure of bladder SMC to NO in culture was found to decrease both DNA and protein synthesis [24] suggesting a role for modulation of NOS activity in bladder remodeling. Interestingly, iNOS expression in bladder SMC correlates with loss of differentiation markers such as SM-MHC [14].

Felsen et al. [25] showed that genetic deletion or pharmacologic inhibition of iNOS diminished obstructioninduced growth of the bladder and attenuated spontaneous detrusor contractions in a murine model of partial bladder outlet obstruction. These findings suggest that actions of NO may underlie certain aspects of pathologic bladder function following obstruction. The effects of $\mathrm{NO}$ on bladder muscle appear to be regional in that NO-induced relaxation of the urethra and bladder outlet has been reported, whereas the effect of NO on detrusor contraction or relaxation remains equivocal. Together, these observations suggest that targeting NO activity may have therapeutic value for ameliorating bladder dysfunction.

\section{Bladder Smooth Muscle-ECM Interactions}

Chronic overdistension of the bladder, as occurs under conditions of outlet obstruction, leads to a fibroproliferative response within the bladder wall, characterized by proliferation of bladder SMC and other cell types, turnover of the ECM and loss of differentiated muscle function. A recent study by Upadhyay et al. [26] demonstrated time-dependent changes in expression of integrins and the ECM constituents type I and type III collagen in the whole rat bladder following distension in vivo and in rat bladder SMC exposed to cyclic stretch-relaxation in vitro. In that study, blockade of integrin function with RGD peptides inhibited stretch-stimulated DNA synthesis in bladder SMC, implicating integrins in regulation of SMC proliferation elicited by mechanical stimulation. In a related study, denaturation of the collagen substrate for bladder SMC cultured in vitro was found to upregulate proliferation compared to the native collagen control [27]. Heat denaturation is believed to mimic the collagen turnover that occurs during ECM remodeling. In that report, induction of SMC proliferation in response to denatured collagen was almost completely inhibited in the presence of PD98059, a pharmacologic inhibitor of ErkMAPK signaling. Collectively, these and other studies highlight the role of the ECM as an active participant in regulating the response of bladder SMC to mechanical stimuli, and not merely an inert support structure. In addition, they suggest that pathologic tissue remodeling within the bladder wall could be mitigated by targeting matrix turnover and integrin-mediated signaling [2830].

\section{Cholesterol Signaling Regulates Urinary Tract Smooth Muscle}

Several recent reports have indicated a role for lipid raft microdomains in regulating growth factor signaling in bladder smooth muscle [7, 31, 32]. Lipid rafts are specialized membrane domains that are enriched in specific lipid components such as cholesterol and sphingolipids, and that are either flat (so-called G domains) or that adopt an invaginated morphology resulting from oligomerization of constituent caveolin proteins (caveolae). These organelles are believed to function as signaling platforms in which interactions between receptor proteins, adapter and effector molecules are facilitated to regulate signal generation, amplification and diversification [33]. Stehr et al. [7] showed that ablation of rafts by depletion of cholesterol inhibited PDGF-stimulated DNA synthesis in urinary tract SMC. Moreover, increasing membrane cholesterol was found to boost basal and PDGF-stimulated DNA synthesis in human bladder SMC [7], whereas treatment with the cholesterol synthesis inhibitor lovastatin attenuated PDGF-stimulated thymidine uptake. TGF $\beta 1$ signaling, in contrast, was negatively regulated by caveolae in bladder SMC [32]. Ablation of caveolae by depletion of membrane cholesterol enhanced the growth inhibitory activity of TGF $\beta 1$ in bladder and ureteral SMC. Consistent with these findings, Woodman et al. [31] observed that mice in which the gene encoding caveolin-1 had been knocked out lacked caveolae and displayed hy- 
pertrophy and diminished contractility of bladder smooth muscle compared to controls. Thus, lipid raft microdomains appear to be important in regulating growth of bladder smooth muscle.

As noted earlier, the Rho-ROCK axis is known to regulate smooth muscle contraction and tone. Rho family members are pos-translationally modified by isoprenylation, i.e. attachment of geranylgeranylpyrophosphate, a lipid intermediate in cholesterol synthesis [34]. This modification leads to plasma membrane targeting and activation of Rho GTPase activity, and can be inhibited by statin drugs [35]. Although the effects of cholesterol manipulation on the lower urinary tract have not been reported, statin-mediated inhibition of Rho isoprenylation has been shown to attenuate proliferation and enhance apoptosis of vascular SMC [35]. Collectively, these observations suggest the intriguing possibility that statinmediated manipulation of cholesterol levels may have therapeutic benefit in conditions characterized by aberrant growth or impaired contractility of bladder smooth muscle.

\section{Summary and Conclusions}

Recent advances in our understanding of the signals that regulate growth and contractility of bladder smooth muscle have begun to identify potential therapeutic targets for the treatment of bladder diseases characterized by aberrant growth and/or contraction of smooth muscle. In the postgenomic era, researchers have access to a wealth of data on protein structure, drug specificity and pharmacokinetic properties, and potential off-target effects. Consequently, this information can be leveraged to design agents with exquisite selectivity for one or more target proteins, thereby minimizing unwanted side effects. The success of agents such as Gleevec and Herceptin suggests that this strategy will bear fruit for treatment of bladder dysfunction.

\section{References}

1 Iqbal J, Zaidi M: Molecular regulation of mechanotransduction. Biochem Biophys Res Commun 2005;328:751-755.

$\checkmark 2$ Yamaguchi O: Response of bladder smooth muscle cells to obstruction: signal transduction and the role of mechanosensors. Urology 2004; 63:11-16.

$\checkmark 3$ Adam RM, Roth JA, Cheng HL, Rice DC, Khoury J, Bauer SB, Peters CA, Freeman MR: Signaling through PI3K/Akt mediates stretch and PDGF-BB-dependent DNA synthesis in bladder smooth muscle cells. J Urol 2003;169: 2388-2393.

4 Tamura I, Rosenbloom J, Macarak E, Chaqour B: Regulation of Cyr61 gene expression by mechanical stretch through multiple signaling pathways. Am J Physiol Cell Physiol 2001;281: C1524-C1532.

5 Adam RM, Eaton SH, Estrada C, Nimgaonkar A, Shih SC, Smith LE, Kohane IS, Bagli D, Freeman MR: Mechanical stretch is a highly selective regulator of gene expression in human bladder smooth muscle cells. Physiol Genomics 2004;20:36-44.

6 Nguyen HT, Adam RM, Bride SH, Park JM, Peters CA, Freeman MR: Cyclic stretch activates p38 SAPK2-, ErbB2-, and AT1-dependent signaling in bladder smooth muscle cells. Am J Physiol Cell Physiol 2000;279:C1155C1167.
7 Stehr M, Adam RM, Khoury J, Zhuang L, Solomon KR, Peters CA, Freeman MR: Platelet derived growth factor-BB is a potent mitogen for rat ureteral and human bladder smooth muscle cells: dependence on lipid rafts for cell signaling. J Urol 2003; 169:1165-1170.

-8 Hu Y, Bock G, Wick G, Xu Q: Activation of PDGF receptor alpha in vascular smooth muscle cells by mechanical stress. FASEB J 1998; 12:1135-1142.

$\checkmark$ Fischer OM, Streit S, Hart S, Ullrich A: Beyond Herceptin and Gleevec. Curr Opin Chem Biol 2003;7:490-495.

10 Kubota Y, Kajioka S, Biers SM, Yokota E, Kohri K, Brading AF: Investigation of the effect of the c-kit inhibitor Glivec on isolated guinea-pig detrusor preparations. Auton Neurosci 2004; 115:64-73.

11 Riento K, Ridley AJ: ROCKS: multifunctional kinases in cell behaviour. Nat Rev Mol Cell Biol 2003;4:446-456.

12 Wibberley A, Chen Z, Hu E, Hieble JP, Westfall TD: Expression and functional role of Rhokinase in rat urinary bladder smooth muscle. Br J Pharmacol 2003; 138:757-766.

13 Bing W, Chang S, Hypolite JA, DiSanto ME, Zderic SA, Rolf L, Wein AJ, Chacko S: Obstruction-induced changes in urinary bladder smooth muscle contractility: a role for Rho kinase. Am J Physiol Renal Physiol 2003;285: F990-F997.
14 Johansson R, Persson K: Phenotypic modulation of cultured bladder smooth muscle cells and the expression of inducible nitric oxide synthase. Am J Physiol Regul Integr Comp Physiol 2004;286:R642-R648.

$>15$ Yoshida T, Sinha S, Dandre F, Wamhoff BR, Hoofnagle MH, Kremer BE, Wang DZ, Olson EN, Owens GK: Myocardin is a key regulator of CArG-dependent transcription of multiple smooth muscle marker genes. Circ Res 2003; 92:856-864.

16 Uehata M, Ishizaki T, Satoh H, Ono T, Kawahara T, Morishita T, Tamakawa H, Yamagami $\mathrm{K}$, Inui J, Maekawa M, Narumiya S: Calcium sensitization of smooth muscle mediated by a Rho-associated protein kinase in hypertension. Nature 1997;389:990-994.

17 Rees RW, Foxwell NA, Ralph DJ, Kell PD, Moncada S, Cellek S: Y-27632, a Rho-kinase inhibitor, inhibits proliferation and adrenergic contraction of prostatic smooth muscle cells. J Urol 2003;170:2517-2522.

18 Austin PF, Cook BL, Niederhoff RA, Manson SR, Coplen DE, Weintraub SJ: Inhibition of mitogenic signaling and induction of apoptosis in human bladder smooth muscle cells treated with doxazosin. J Urol 2004; 172:1662-1665. 
19 Benning CM, Kyprianou N: Quinazoline-derived alpha-1-adrenoceptor antagonists induce prostate cancer cell apoptosis via an alpha-1adrenoceptor-independent action. Cancer Res 2002;62:597-602.

-20 Kushida N, Kabuyama Y, Yamaguchi O, Homma Y: Essential role for extracellular $\mathrm{Ca}^{2+}$;in JNK activation by mechanical stretch in bladder smooth muscle cells. Am J Physiol Cell Physiol 2001;281:C1165-C1172.

-21 Nozaki K, Tomizawa K, Yokoyama T, Kumon $\mathrm{H}$, Matsui $\mathrm{H}$ : Calcineurin mediates bladder smooth muscle hypertrophy after bladder outlet obstruction. J Urol 2003;170:2077-2081.

-22 Stothers L, Laher I, Christ GT: A review of the $L$-arginine-nitric oxide-guanylate cyclase pathway as a mediator of lower urinary tract physiology and symptoms. Can J Urol 2003; 10: 1971-1980.

-23 Johansson RK, Poljakovic M, Andersson KE, Persson K: Expression of nitric oxide synthase in bladder smooth muscle cells: regulation by cytokines and $L$-arginine. J Urol 2002;168: 2280-2285.

-24 Johansson R, Pandita RK, Poljakovic M, Garcia-Pascual A, De Vente J, Persson K: Activity and expression of nitric oxide synthase in the hypertrophied rat bladder and the effect of nitric oxide on bladder smooth muscle growth. J Urol 2002;168:2689-2694.
25 Felsen D, Dardashti K, Ostad M, Lemer ML, Gross SS, Chen J, Vaughan ED Jr, Poppas DP Inducible nitric oxide synthase promotes pathophysiological consequences of experimental bladder outlet obstruction. J Urol 2003; 169:1569-1572.

26 Upadhyay J, Aitken KJ, Damdar C, Bolduc S, Bagli DJ: Integrins expressed with bladder extracellular matrix after stretch injury in vivo mediate bladder smooth muscle cell growth in vitro. J Urol 2003;169:750-755.

27 Herz DB, Aitken K, Bagli DJ: Collagen directly stimulates bladder smooth muscle cell growth in vitro: regulation by extracellular regulated mitogen activated protein kinase. J Urol 2003;170:2072-2076.

28 Matsuno H, Stassen JM, Vermylen J, Deckmyn $\mathrm{H}$ : Inhibition of integrin function by a cyclic RGD-containing peptide prevents neointima formation. Circulation 1994;90:22032206.

29 Roy J, Kazi M, Hedin U, Thyberg J: Phenotypic modulation of arterial smooth muscle cells is associated with prolonged activation of ERK1/2. Differentiation 2001;67:50-58.

30 Matsuno H, Ishisaki A, Nakajima K, Kozawa $\mathrm{O}$ : Effect of a synthetic matrix metalloproteinase inhibitor (ONO-4817) on neointima formation in hypercholesterolemic hamsters. J Cardiovasc Pharmacol 2004;44:57-65.
31 Woodman SE, Cheung MW, Tarr M, North AC, Schubert W, Lagaud G, Marks CB, Russell RG, Hassan GS, Factor SM, Christ GJ, Lisanti MP: Urogenital alterations in aged male caveolin-1 knockout mice. J Urol 2004; 171:950957.

32 Stehr M, Estrada CR, Khoury J, Danciu TE, Sullivan MP, Peters CA, Solomon KR, Freeman MR, Adam RM: Caveolae are negative regulators of transforming growth factor-beta 1 signaling in ureteral smooth muscle cells. J Urol 2004; 172:2451-2455.

33 Simons K, Toomre D: Lipid rafts and signal transduction. Nat Rev Mol Cell Biol 2000;1: 31-39.

34 Hirai A, Nakamura S, Noguchi Y, Yasuda T, Kitagawa M, Tatsuno I, Oeda T, Tahara K, Terano T, Narumiya S, Kohn LD, Saito Y: Geranylgeranylated rho small GTPase(s) are essential for the degradation of $\mathrm{p} 27 \mathrm{Kip} 1$ and facilitate the progression from $\mathrm{G} 1$ to $\mathrm{S}$ phase in growth-stimulated rat FRTL-5 cells. J Biol Chem 1997;272:13-16.

35 Takemoto M, Liao JK: Pleiotropic effects of 3-hydroxy-3-methylglutaryl coenzyme a reductase inhibitors. Arterioscler Thromb Vasc Biol 2001;21:1712-1719. 